Effective Interactions and

Operators in Nuclei within the

No-Core Shell Model

B.R. Barrett, Petr Navratil, I. Stetcu, J.P. Vary

September 16, 2005

12th International Conference on Capture Gamma-Ray Spectroscopy and RElated Topics

Notre Dame, IN, United States

September 4, 2005 through September 9, 2005 
This document was prepared as an account of work sponsored by an agency of the United States Government. Neither the United States Government nor the University of California nor any of their employees, makes any warranty, express or implied, or assumes any legal liability or responsibility for the accuracy, completeness, or usefulness of any information, apparatus, product, or process disclosed, or represents that its use would not infringe privately owned rights. Reference herein to any specific commercial product, process, or service by trade name, trademark, manufacturer, or otherwise, does not necessarily constitute or imply its endorsement, recommendation, or favoring by the United States Government or the University of California. The views and opinions of authors expressed herein do not necessarily state or reflect those of the United States Government or the University of California, and shall not be used for advertising or product endorsement purposes. 


\title{
Effective Interactions and Operators in Nuclei within the No-Core Shell Model
}

\author{
Bruce R. Barrett ${ }^{*}$, Petr Navratil ${ }^{\dagger}$, Ionel Stetcu* ${ }^{*}$ and James P. Vary ${ }^{\dagger, * *, *}$ \\ ${ }^{*}$ Department of Physics, University of Arizona, P.O. Box 210081, Tucson, Arizona 85721 \\ ${ }^{\dagger}$ Lawrence Livermore National Laboratory, Livermore, P.O. Box 808, California 94551 \\ ${ }^{*}$ Department of Physics and Astronomy, Iowa State University, Ames, Iowa 50011 \\ ${ }^{\ddagger}$ SLAC, MS 81, 2575 Sand Hill Rd, Menlo Park, California 94025
}

\begin{abstract}
We review the application of effective operator formalism to the ab initio no core shell model (NCSM). For short-range operators, such as the nucleon-nucleon potential, the unitarytransformation method works extremely well at the two-body cluster approximation and good results are obtained for the binding energies and excitation spectra of light nuclei $(A \leq 16)$. However, for long-range operators, such as the radius or the quadrupole moment, performing this unitary transformation at the two-body cluster level, does not include the higher-order correlations needed to renormalize these long-range operators adequately. Usually, such correlations can be obtained either by increasing the order of the cluster approximation, or by increasing the model space. We will discuss the difficulties of these approaches as well as alternate possible solutions for including higher-order correlations in small model spaces.
\end{abstract}

Keywords: shell model, nuclear spectra, electromagnetic transitions

PACS: 21.60.Cs, 23.20.-g, 23.20.Js

\section{INTRODUCTION}

In the last few years, significant advances in theoretical methods and computer power have allowed the description of the low-lying states in light nuclei from first principles, i.e., starting from realistic nucleon-nucleon $(\mathrm{NN})$ interactions that fit the experimental phase-shifts [1], and theoretical three-body forces [2]. The no-core shell model (NCSM) is a particularly flexible method that allows all the $A$ nucleons to interact and preserves all the symmetries of the original Hamiltonian [3, 4]. The main tool is a unitary transformation [5, 6] of the Hamiltonian from the infinite space to a model space, which allows a diagonalization in a many-body basis. In the lowest cluster approximation, the unitary transformation approach works extremely well for binding energies and excitation spectra of light $(A \leq 16)$ and selected medium-mass nuclei [7].

Besides spectra, other properties of the nuclear states are of interest, as they impose a strong test on the theoretical wave functions. In particular, we concentrate on electromagnetic transitions. The same unitary transformation used to compute the effective interaction can be used in order to obtain effective operators in the model spaces used to diagonalize the effective Hamiltonian. We found that, in the lowest approximation, the unitary transformation has little effect on long-range observables [8], such as quadrupole moments and radii. In retrospective, these results can be understood as long-range observables "feel" effects due essentially to all $A$ nucleons; the two-body cluster approximation, however, accounts for short-range correlations and cannot accommodate 
long-range correlations.

In this paper, we shortly review the theory of effective operators in the framework of the unitary transformation approach and discuss applications to light nuclei, as well as possible avenues for including more higher-order correlations in small model spaces.

\section{THEORETICAL APPROACH}

For over fifty years, the state of the art method for a microscopic description of nuclear states has been the phenomenological shell model, where on top of the single particle states one adds a residual interaction and diagonalizes in a restricted model space, assuming an inert core. Work by Arima and Horie [9] and later extended by Bertsch [10] introduced the core polarization concept into the nuclear shell model. Core polarization is a method to induce long-range correlations in a nucleus, and its effect was an improved agreement of the nuclear spectrum with the experiment. Without entering into details concerning issues like convergence, we have to point out that, while reasonable results have been obtained for the nuclear spectra, applications of the same many-body techniques to other observables, such as the magnetic dipole and electric quadrupole, never achieved the same level of success. In particular, the empirical effective charges of $1.5 e$ for the proton and $0.5 e$ for the neutron have remained a puzzle for decades.

In the NCSM, we use a unitary transformation in order to include correlations left out by the truncation of the model space. The transformed Hamiltonian

$$
\mathscr{H}=e^{-S} H e^{S},
$$

preserves the spectrum of the initial Hamiltonian. Moreover, if the antihermitian operator $S$ is determined by the condition $P \mathscr{H} Q=0$, that is, the transformed Hamiltonian does not couple the model, or $P$, space with the excluded, or $Q$, space, then the effective interaction in the model space is energy independent. Furthermore, if one determines the operator $S$ so that the additional decoupling condition $Q \mathscr{H} P=0$ is fulfilled, it can be shown that the effective operators determined by the transformation

$$
\mathscr{O}=e^{-S} O e^{S}
$$

are also energy independent $[5,11]$. Formally, the operator $S$ can be written by means of another operator $\omega$ as $S=\operatorname{arctanh}\left(\omega-\omega^{\dagger}\right)$, where the new operator fulfills $Q \omega P=\omega$. Hence, one obtains the energy-independent effective Hamiltonian in the model space $P$

$$
H_{e f f}=P \mathscr{H} P=\frac{P+P \omega^{\dagger} Q}{\sqrt{P+\omega^{\dagger} \omega}} H \frac{P+Q \omega P}{\sqrt{P+\omega^{\dagger} \omega}},
$$

and, analogously, any observable can be transformed to the $P$ space as $[5,11]$

$$
O_{e f f}=P \mathscr{O} P=\frac{P+P \omega^{\dagger} Q}{\sqrt{P+\omega^{\dagger} \omega}} O \frac{P+Q \omega P}{\sqrt{P+\omega^{\dagger} \omega}} .
$$

The transformed operator preserves the same symmetries of the initial operator, e.g., the tensor character, as the transformation is a spin-isospin scalar. However, we have to 
TABLE 1. $B(E 2)$, in $e^{2} \mathrm{fm}^{4}$, and relative kinetic energy expectation value, in $M e V$, for selected nuclei and model spaces, using the bare and effective operators computed in the two-body cluster approximation.

\begin{tabular}{ccccc}
\hline Nucleus & Observable & Model Space & Bare operator & Effective operator \\
\hline${ }^{6} \mathrm{Li}$ & $B\left(E 2,1^{+} 0 \rightarrow 3^{+} 0\right)$ & $2 \hbar \Omega$ & 2.647 & 2.784 \\
\hline${ }^{6} \mathrm{Li}$ & $B\left(E 2,1^{+} 0 \rightarrow 3^{+} 0\right)$ & $10 \hbar \Omega$ & 10.221 & - \\
\hline${ }^{6} \mathrm{Li}$ & $B\left(E 2,2^{+} 0 \rightarrow 1^{+} 0\right.$ & $2 \hbar \Omega$ & 2.183 & 2.269 \\
\hline${ }^{6} \mathrm{Li}$ & $B\left(E 2,2^{+} 0 \rightarrow 1^{+} 0\right.$ & $10 \hbar \Omega$ & 4.502 & - \\
\hline${ }^{10} \mathrm{C}$ & $B\left(E 2,2_{1}^{+} 0 \rightarrow 0^{+} 0\right)$ & $4 \hbar \Omega$ & 3.05 & 3.08 \\
\hline${ }^{12} \mathrm{C}$ & $B\left(E 2,2_{1}^{+} 0 \rightarrow 0^{+} 0\right)$ & $4 \hbar \Omega$ & 4.03 & 4.05 \\
\hline${ }^{4} \mathrm{He}$ & $\left\langle\right.$ g.s. $\left|T_{\text {rel }}\right|$ g.s. $\rangle$ & $8 \hbar \Omega$ & 71.48 & 154.51 \\
\hline
\end{tabular}

point out that the transformation is much more involved for a non-scalar observable than for a scalar one. Indeed, because general tensor operators can couple different angular momentum states, one has to use different transformation operators $\omega$ in Eq. (4).

Once one obtains $\omega$, the problem is solved. However, this is a task as difficult as solving the full $A$-body problem, as the exact solution is an $A$-body operator, regardless of the rank of the interaction with which one starts. Therefore, one makes the cluster approximation, in which one finds $\omega$ for the problem involving $a$ nucleons $(a<A)$, and then uses this solution to compute the effective Hamiltonian and operators in the model space via Eqs. (3) and (4). This interaction, which reproduces exactly the spectrum of the $a$-body problem, is then used to compute the solution to the $A$-body problem. There are two important convergence properties. Thus, keeping the model space fixed and increasing the cluster size, one converges to the exact solution. The same convergence to the exact solution can be also obtained by keeping the cluster size fixed and increasing the model spaces. In principle, because of these properties one expects to observe larger renormalization in smaller model spaces and for small cluster sizes, or, in other words, the renormalization effects are more visible in small model spaces for a given cluster approximation.

\section{RESULTS AND DISCUSSION}

In a recent publication [8], we have investigated the effect of the renormalization of electromagnetic operators in realistic calculations for $p$-shell nuclei. Because our first goal was a qualitative understanding of the influence of effective operators and not a highly accurate description of the experimental data, we have used only two-body, high precision NN interactions, leaving out the three-body forces for the time being. This also motivates the use of rather small model spaces and of the two-body cluster approximation, given that, as expected from the convergence properties of effective operators mentioned at the end of the last section, in such conditions, larger renormalization effects are expected.

In calculations of several nuclei, we saw very little effect of the effective operators 
in the results for the $B(E 2)$, as illustrated in Table 1 . One may find this somehow surprising, as previous investigations in the framework of the NCSM [12] successfully obtained the correct effective proton and neutron phenomenological charges, which haunted the nuclear structure community for so long. However, the main difference is that the calculation in Ref. [12] included up to six-body correlations in the case of ${ }^{6} \mathrm{Li}$, i.e., the nucleus chosen for this investigation. Comparison of the two results already suggests that higher-order clusters can play an important role in the renormalization of the $E 2$ operator.

In contrast with the $E 2$ operator, the kinetic energy operator is well renormalized at the two-body cluster level, as seen in Table 1. The difference can be understood in terms of the character of the two operators. The kinetic energy is short range, while the quadrupole is long range; at the two-body cluster level, the unitary transformation renormalizes mainly the short-range core of the interaction, leaving unchanged the long range part. Hence, in order to account for long-range correlations in the two-body cluster approximation, one needs to enlarge the model space; an illustration of this approach can be seen in Table 1 , where the $B(E 2)$ value obtained in $10 \hbar \Omega$ is significantly larger than the one calculated in $2 \hbar \Omega$. Although for very light nuclei increasing the model spaces is not a problem, the use of a large model space quickly becomes numerically intractable for heavier systems. Because the utilization of very large model spaces is impractical, if not impossible, for all but the lightest nuclei $(A \leq 10)$ it is worthwhile to develop new methods to introduce more correlations in a smaller model space. Such methods should also provide a better understanding of the underlying physics. Possible such methods would include, among others,

1. performing large model-space calculations for light nuclei, and then explicitly truncating these results into a very small model space, as done in Ref. [12] for ${ }^{6} \mathrm{Li}$, thereby building into the final results all the $A$-body correlations, and

2. calculating the effective Hamiltonian in a large model space and using its matrix elements as input into a many-body perturbation theory calculation of a new, further renormalized effective Hamiltonian in a much smaller model space.

Work is in progress to implement such approaches.

\section{ACKNOWLEDGMENTS}

B.R.B and I.S. acknowledge partial support by NFS grant PHY0244389. The work was performed in part under the auspices of the U. S. Department of Energy by the University of California, Lawrence Livermore National Laboratory under contract No. W-7405Eng-48. P.N. received support from LDRD contract 04-ERD-058. J.P.V. acknowledges partial support by USDOE grant No DE-FG-02-87ER-40371.

\section{REFERENCES}

1. R. B. Wiringa, V. G. J. Stoks and R. Schiavilla, Phys. Rev. C 51, 38 (1995);R. Machleidt, F. Sammarruca and Y. Song, Phys. Rev. C 53, R1483 (1996). 
2. S. A. Coon, M. D. Scadron, P. C. McNamee, B. R. Barrett, D. W. E. Blatt, and B. H. J. McKeller, Nucl. Phys. A317, 242 (1979); S.A. Coon and H. K. Han, Few Body Systems 30, 131 (2001).

3. P. Navrátil, J. P. Vary, W. E. Ormand, and B. R. Barrett, Phys. Rev. Lett. 87, 172502 (2001).

4. P. Navrátil, J. P. Vary, and B. R. Barrett, Phys. Rev. Lett. 84, 5728 (2000); Phys. Rev. C 62, 054311 (2000).

5. S. Okubo, Prog. Theor. Phys. 12, 603 (1954).

6. J. Da Providencia and C. M. Shakin, Ann. of Phys. 30, 95 (1964); K. Suzuki and S. Y. Lee, Prog. Theor. Phys. 64, 2091 (1980); K. Suzuki, Prog. Theor. Phys. 68, 246 (1982); K. Suzuki and R. Okamoto, Prog. Theor. Phys. 70, 439 (1983); K. Suzuki, Prog. Theor. Phys. 68, 1999 (1982); K. Suzuki and R. Okamoto, Prog. Theor. Phys. 92, 1045 (1994).

7. S. Popescu, S. Stoica, J.P. Vary and P. Navratil, to be published.

8. I. Stetcu, B. R. Barrett, P. Navrátil, and J. P. Vary, Phys. Rev. C 71, 044325 (2005).

9. A. Arima and H. Horie, Prog. Theor. Phys. (Kyoto) 12, 623 (1954); Phys. Rev. 99,778 (1955).

10. G. F. Bertsch, Nucl. Phys. 74, 234 (1965).

11. P. Navrátil, H. Geyer, and T. T. S. Kuo, Phys. Lett. B 315, 1 (1993).

12. P. Navrátil, M. Thoresen, and B. R. Barrett, Phys. Rev. C 55, R573 (1997). 